\title{
The role of working memory in attentional capture
}

\author{
NILLI LAVIE \\ University College London, London, England \\ and \\ JAN DE FOCKERT \\ Goldsmiths College, University of London, London, England
}

\begin{abstract}
Much previous research has demonstrated that visual search is typically disrupted by the presence of a unique "singleton" distractor in the search display. Here we show that attentional capture by an irrelevant color singleton during shape search critically depends on availability of working memory to the search task: When working memory is loaded in a concurrent yet unrelated verbal short-term memory task, capture increases. These findings converge with previous demonstrations that increasing working memory load results in greater distractor interference in Stroop-like tasks (de Fockert, Rees, Frith, \& Lavie, 2001; Lavie, Hirst, de Fockert, \& Viding, 2004), which support the hypothesis that working memory provides goal-directed control of visual selective attention allowing to minimize interference by goal-irrelevant distractors.
\end{abstract}

Coherent goal-directed behavior requires top-down control of attention, so that attention is allocated to goalrelevant stimuli rather than to goal-irrelevant distractors. Executive cognitive control functions that are typically associated with the frontal lobe, such as working memory, have long been thought to play a major role in such goaldirected control of attention (Baddeley, 1996; Desimone $\&$ Duncan, 1995). However, although neuropsychological studies have generally implicated the frontal lobe in selective attention, behavioral evidence for a causal role for working memory in goal-directed control of visual selective attention has been rather scarce. ${ }^{1}$

In a series of studies, Logan (1978) failed to find any effect of verbal working memory load on efficiency of search as expressed in the slopes of search set size functions. Woodman, Vogel, and Luck (2001) failed to find any effect of visual working memory load on the efficiency of search performance. More recently, Woodman and Luck (2004) found an effect of spatial memory load on efficiency of search. This, however, may be attributed to the shared content between the tasks used (the search task required to specify the location of a target attribute) rather than to higher level, content-independent cognitive control of visual search by working memory.

More positive suggestions of a cognitive control role come from studies showing that individual differences in working memory span correlate with performance in

This research was supported by an MRC grant to the first author. Correspondence related to this article should be addressed to N. Lavie, Department of Psychology, University College London, Gower Street, London WC1E 6BT, England (e-mail: n.lavie@ucl.ac.uk).
Stroop-like paradigms. For example, Kane and Engle (2003) showed that low-span subjects make more erroneous responses to a distracting incongruent word in the Stroop task than high-span subjects, suggesting involvement of working memory capacity in control of distractor responses. However, because these findings are correlative, they cannot inform about any causal role of working memory in attention. It is possible, for example, that better selective attention ability allows for better performance in working memory tasks.

Working memory was found to play a causal role in determining negative priming (NP) by distractors that are subsequently presented as targets (Engle, Conway, Tuholski, \& Shisler, 1995). Engle et al. presented a word after every pair of prime-probe trials and found that NP from prime distractors was reduced with more than one word to remember. It is not clear, however, whether high working memory load reduced inhibition of distractors (an effect on selective attention) or reduced their encoding into memory and the retrieval of previous episodes (both effects on long-term memory).

The first clear evidence for a causal role of working memory in control of interference by irrelevant visual distractors has been provided in a series of studies by Lavie and colleagues (de Fockert, Rees, Frith, \& Lavie, 2001; Lavie, 2000; Lavie, Hirst, de Fockert, \& Viding, 2004). Lavie and colleagues have suggested that working memory serves to actively maintain processing priorities, specifying which stimuli are currently task relevant and which are irrelevant. From this claim, it follows that reducing availability of working memory for a selective attention task (by loading working memory in a concurrent, yet unrelated task) should result in reduced efficiency of 
focusing attention on the relevant stimuli, with greater interference by irrelevant distractors.

This prediction received support from behavioral experiments that showed that flanker effects in a selective attention task (consisting of a central target letter and a flanking distractor letter) were significantly increased under conditions of high working memory load (in which subjects were required to rehearse a set of six digits) in comparison with low working memory load (in which there was one digit to rehearse) during performance of the attention task (Lavie et al., 2004). A functional imaging experiment, in which working memory load was manipulated during performance of a face-name, Stroop-like task, also showed increased interference by incongruent distractor faces as well as increased neural activity related to the presence of a distractor face in face-selective areas of visual cortex with high working memory load (de Fockert et al., 2001). Although the convergence of behavioral and neuroimaging results makes an appealing case for a causal role of working memory in preventing interference by goal-irrelevant distractors, so far positive evidence has come only from Stroop-like congruency tasks. By contrast, as we discussed earlier, visual search studies failed to find any effects of working memory load on the efficiency of distractor rejection in an unrelated search task.

Our purpose in the present study was, therefore, to examine whether working memory has a causal role in distractor rejection during visual search. We reasoned that the discrepancy in results between search and Stroop-like tasks may be due to the following: The distractor in Strooplike tasks is typically a strong competitor for response selection. Its rejection may, therefore, tax high-level cognitive control functions, such as working memory. However, the nontarget stimuli in typical search tasks do not include any particularly strong competitor for selection, and the competition between target and nontargets can typically be resolved at early perceptual levels (e.g., determined by target-nontarget similarity; Duncan \& Humphreys, 1989). Thus, rejecting them does not require higher level cognitive control.

It follows that if one of the search items is a strong competitor for selection, as is the case when an odd "singleton" distractor pops out more easily than the target and thus captures attention (for review, see Yantis, 2000), then rejection of that competing distractor should depend on availability of working memory to provide goal-directed control of the search task. We therefore predicted that the capture of attention by an irrelevant singleton during visual search would be increased by high (vs. no or low) working memory load. Testing this prediction would allow us not only to examine whether working memory has a general role in selective attention that can generalize across very different measures of distractor processing, ${ }^{2}$ but also to shed some light on a controversy in the study of attentional capture about whether it is purely stimulus driven (i.e., determined only by the relative salience of target and singleton; Theeuwes, 1996) or whether atten- tional capture may also be subject to top-down control (see, e.g., Bacon \& Egeth, 1994).

\section{EXPERIMENT 1}

In Experiment 1, we compared capture by an irrelevant singleton during visual search between a single-task condition and a dual-task condition in which the search task was combined with a working memory task. The search task was adapted from Theeuwes (1992). Subjects were required to search for a circle among diamonds and make a speeded response to the orientation of a line within it. An irrelevant color singleton was present in some trials and absent in others. Attentional capture was assessed by the extent to which target response times (RTs) were slower in the presence of the singleton. In the dual-task condition, subjects were requested to rehearse a set of six digits during the search task in order to determine whether a memory probe following the search task was present or absent in the memory set of that trial.

If availability of working memory for the search task determines attentional capture by an irrelevant singleton, then singleton interference should be greater under the dual-task (involving high working memory load) condition than under the single-task condition (involving no working memory load).

\section{Method}

Subjects. Twelve paid students from University College London (UCL) participated. The subjects in all the experiments reported had normal or corrected-to-normal vision and normal color vision.

Stimuli and Apparatus. We used a computer running MEL software connected to a 15 -in. SVGA monitor to run the experiment. Viewing distance was fixed at $60 \mathrm{~cm}$ with a custom-built viewing box plus hood. The memory set in the dual-task condition consisted of six digits, chosen at random with no repetition from 1 to 9 with the constraints that no more than three digits could be in ascending or descending order. The memory probe consisted of a single digit and a question mark (e.g., "5?"). Each digit occurred equally often in the memory sets and served equally often as the memory probe. Each digit subtended $0.4^{\circ}$ horizontally and $0.6^{\circ}$ vertically. The whole set subtended $2.0^{\circ}$ to the left and right of fixation.

The search displays consisted of a circle of nine shapes equally spaced. The circle radius was $3.4^{\circ}$ from fixation to the center of each shape. Target shape was a circle of a diameter of $1.4^{\circ}$. Nontarget shape was a diamond subtending $1.4^{\circ}$ diagonally. A gray line $0.5^{\circ}$ long was positioned in the center of each shape. These lines were either vertical or horizontal when presented in the target or were tilted by $22^{\circ}$ to either side of the horizontal or vertical plane when presented in the nontarget shapes. Tilt direction was randomly allocated to each shape.

The singleton shape was green, all other shapes were red, and the background was black. The various conditions of target line tilt, target position, singleton presence and position, and all their combinations, occurred equally often in each block. Four blocks of 72 different displays were created according to these specifications.

Procedure. In the dual-task condition, each trial began with a 500-msec central fixation dot, followed by a memory set present for $1,500 \mathrm{msec}$. The subjects were instructed to memorize the digits in the memory set, because at the end of the trial, they would have to decide whether a probe digit was present in the set. Following the memory set, a fixation dot was presented for $2 \mathrm{sec}$ (to allow subjects 
to rehearse the digit set at least once). In the single-task condition, the initial fixation dot and memory set were not presented. Instead, the trial started with a $2 \mathrm{sec}$ fixation dot. In both task conditions, the 2 -sec fixation display was followed by a 200 -msec search display. The subjects were instructed to use their right hand and press " 0 " on the numeric keypad if the line in the circle was horizontal and " 2 " if it was vertical, as quickly and accurately as possible. In the dualtask condition, the search task was followed by a memory probe. The subjects were instructed to indicate as accurately as possible whether the memory probe was present in the memory set at the start of the trial by pressing with their left hand "q" for "yes" and "w" for "no." The probe was presented until a response was made or $3 \mathrm{sec}$ had elapsed. In the single-task condition, the search task was followed by an asterisk presented for $1,000 \mathrm{msec}$. The fixation dot for the next trial appeared after an intertrial interval of $950 \mathrm{msec}$ in the single-task condition and $750 \mathrm{msec}$ in the dual-task condition. Incorrect responses in either task were followed by a tone. Each subject performed eight blocks of 72 trials, preceded by two practice blocks of 24 trials each, alternating between single- and dual-task conditions, with block order counterbalanced across subjects.

\section{Results}

Memory task. Mean error rate to the memory probe was $14 \%$, mean probe RT was $1,038 \mathrm{msec}$.

Search task. Figure 1 presents the mean RT in the search task as a function of the experimental variables. Trials with incorrect responses in either the search task or the memory task, or with RTs over $2 \mathrm{sec}$, were excluded from the RT analysis in all the experiments reported. Within-subjects ANOVAs were conducted on the RTs and on the errors with the factors of task (single, dual) and singleton condition (present, absent). In line with previous attentional capture results, the RT ANOVA revealed that RTs were significantly slower in singleton-present than in singletonabsent conditions $[F(1,11)=19.58, p<.01]$. There was no main effect of task on the search RTs $[F(1,11)=3.86$, $p=.075]$. Importantly, a significant interaction between task and singleton condition $[F(1,11)=8.18, p<.02]$ indicated that the singleton effect in the single-task condition $[t(11)=2.16, p=.027$, one-tailed] was increased significantly under the dual-task condition, as we predicted (Figure 1).

The overall error rate on the attention task was low $(M=3 \%)$, with little variation between experimental conditions. The only trend approaching marginal significance was for a greater singleton effect in the dualtask condition ( $1 \%$ increase in errors in the presence of a singleton) than in the single-task condition ( $0 \%$ singleton effect) $[F(1,11)=4.28, p=.06]$, in line with the RTs.

These results thus support our hypothesis that attentional capture by task-irrelevant singletons in a visual search task depends on the availability of working memory for the selective attention task. Subjects suffered from greater interference by an irrelevant singleton during search when they had to rehearse a set of digits in a concurrent working memory task, consistent with this hypothesis.

\section{EXPERIMENT 2}

In Experiment 2, we sought to replicate the effect of working memory load on attentional capture with a different manipulation of working memory load. In this experiment, the subjects always performed the visual search task under dual-task conditions. The search task was interleaved with the "successor naming" working memory task that required recall of digit order. A fourdigit memory set was presented at the start of each trial, and a single memory probe followed the search task at the end of each trial. Subjects were required to indicate which digit followed the memory probe digit in the memory set of each trial. In the high working memory load condition, the digits in the memory set were presented in a random order on each trial. In the low working memory load condition, the digits in the memory set were always in the same order (memory probe digits still differed between trials). The procedure for the search task was the same as in Experiment 1.

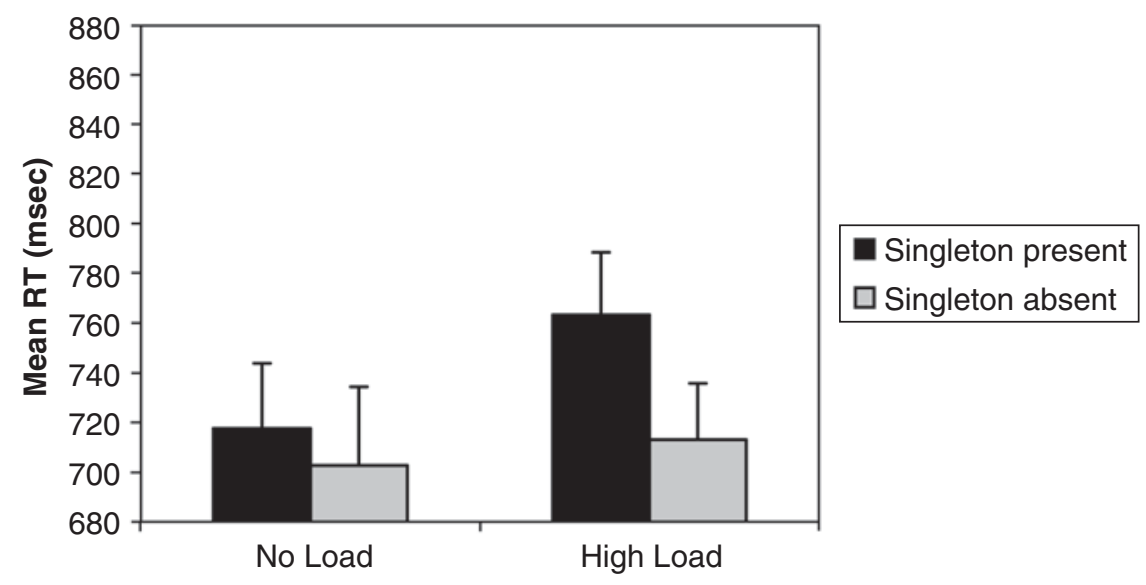

Figure 1: Mean correct response time (RT, in milliseconds) as a function of task condition and singleton condition for Experiment 1. Error bars represent the standard errors of the mean. 


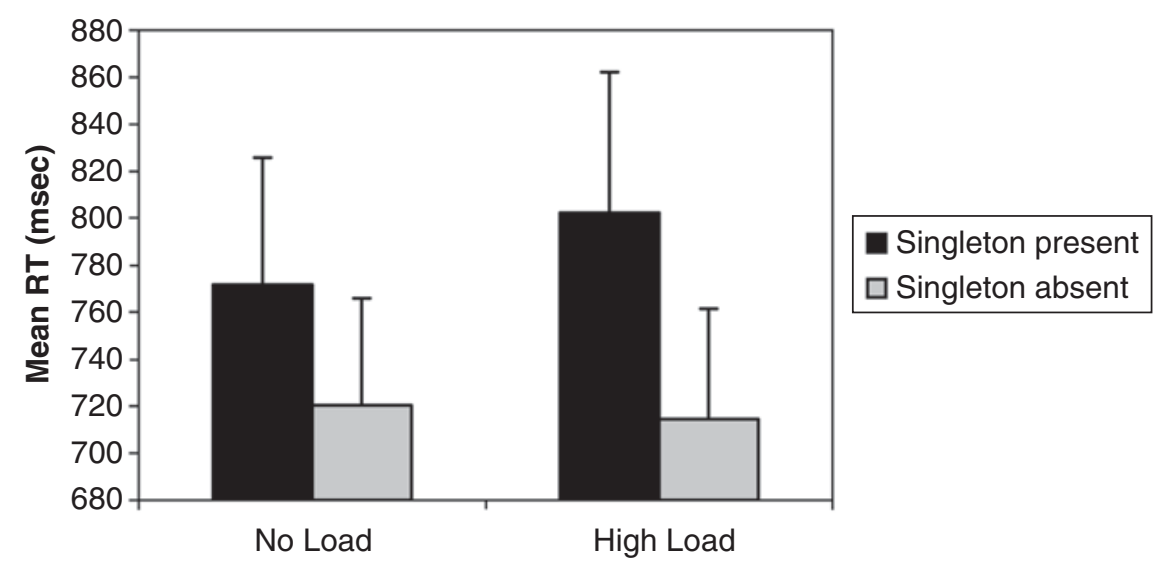

Figure 2: Mean correct response time (RT, in milliseconds) as a function of working memory load and singleton condition for Experiment 2. Error bars represent the standard errors of the mean.

\begin{abstract}
Method
Subjects. The subjects were 12 paid students from UCL who had not participated in the previous experiment.

Stimuli and Procedure. The search task was the same as in Experiment 1 . The changes to the memory task were as follows: A memory set consisting of the digits $0,1,2,3$, and 4 was presented for $1,500 \mathrm{msec}$ at the start of each trial, centered at fixation. In the condition of low working memory load, the memory set was always in order, 01234. In the condition of high working memory load, the digit 0 remained at the start of the memory set, but the order of the digits 1-4 was varied at random in each trial. The memory set was followed by a 1.5 -sec fixation interval in the condition of low load and a 2-sec fixation interval in the condition of high load. Next, the search display was presented for $200 \mathrm{msec}$ as was done in Experiment 1 . Following the search response, a memory probe appeared and remained on the screen until a response was made or until $3 \mathrm{sec}$ had elapsed, whichever occurred first. The memory probe had one digit at fixation that was equally likely to be $0,1,2$, or 3 in the low working memory load condition or $0,1,2,3$, or 4 in the high working memory load condition. Subjects were instructed to key in the digit that followed the memory probe digit in the memory set of that trial. All the positions in the memory set were equally likely to be probed. Conditions of working memory load were blocked again, and all other aspects of procedure were the same as in Experiment 1.
\end{abstract}

\section{Results}

Memory task. Mean RTs were significantly slower with high working memory load $(M=1,152 \mathrm{msec})$ than with low working memory load $[M=743 \mathrm{msec} ; F(1,11)=$ $163, p<.001]$. Error rates were also higher with high working memory load $(M=7 \%)$ than with low working memory load $[M=3 \% ; F(1,11)=6.86, p<.025]$. This confirmed that our manipulation of working memory load was effective.

Search task. Again, the RT ANOVA revealed that RTs were significantly slower in singleton-present than singleton-absent condition $[F(1,11)=21.21, p<.01]$, suggesting that the singleton captured attention. Once again, working memory load had no main effect on search RTs $[F(1,11)=1.85, p=.20]$, but interacted with singleton condition $[F(1,11)=7.71, p<.02]$, showing again that the singleton effect in the low working memory load $[t(11)=3.59, p<.01$, one tailed $]$ was increased under high working memory load, as we predicted (see Figure 2). Error rates were again low $(M=4.75 \%)$, showing a $2.5 \%$ trend for singleton interference $[F(1,11)=2.92$, $p=.10 ; F<1$ for all other comparisons].

In conclusion, Experiment 2 again demonstrates the effect of working memory load on interference by an irrelevant singleton on visual search, thus providing further support for the hypothesis that working memory serves to prevent attention from being captured by an irrelevant singleton distractor.

\section{EXPERIMENT 3}

The manipulation of working memory load in the previous experiments may have involved an increase in the demand on task switching. Although in Experiment 2 the subjects performed the memory task under both conditions of load, they did not need to actively maintain the memory set in the low-load conditions and therefore did not need to actively switch away from the memory set to the search task on each trial. Because coordination of task switching can in itself increase interference by an irrelevant distractor (Lavie et al., 2004), we sought to further confirm in Experiment 3 that load on working memory per se can increase attentional capture. To ensure that subjects have to actively memorize the memory set in both conditions of load, we manipulated working memory load by varying the memory set size. The high-load condition was presented exactly as in Experiment 2; however, in the low-load condition, the memory set contained one digit that was different in each trial. Following the search task, subjects had to key in the successor digit to that presented in the memory set (" 4 " in response to " 3 "). In this way, subjects had to switch from the memory task to the search task in both conditions of working memory load.

\section{Method}

Subjects. The subjects were 10 paid Goldsmiths College students.

Stimuli and Procedure. These were the same as in Experiment 2, except for the presentation of a single digit (randomly selected from 


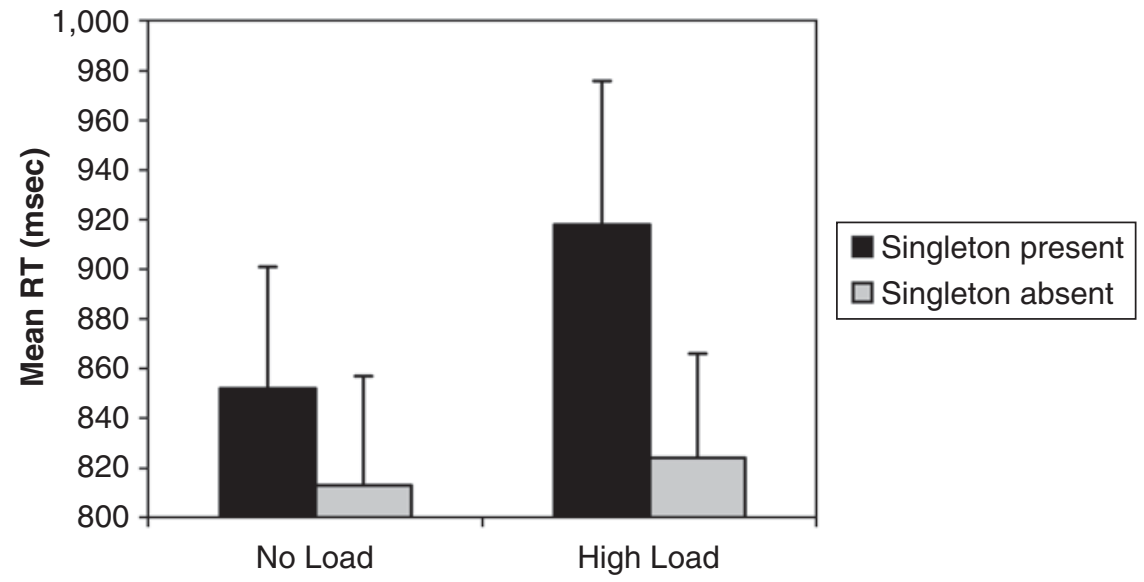

Figure 3: Mean correct response time (RT, in milliseconds) as a function of working memory load and singleton condition for Experiment 3. Error bars represent the standard errors of the mean.

$0,1,2$, and 3 , on each trial) in the memory set and an asterisk as the memory probe (to which the subjects responded by keying in the digit that followed the memory-set digit in ascending order) in the low-load condition.

\section{Results}

Memory task. Mean RTs under high working memory load $(M=1,242 \mathrm{msec})$ were significantly longer $[F(1,9)=203.28, p<.001]$ and error rates $(7 \%)$ significantly higher, $[F(1,9)=9.19, p<.02]$ than under low working memory (mean RT $=226 \mathrm{msec}$, mean error rate $=4 \%$ ).

Search task. Target RT was again slower in singletonpresent than singleton-absent conditions $[F(1,9)=36.39$, $p<.001]$. Working memory load again had no main effect $[F(1,9)=3.10, p>.10]$, but interacted with singleton condition $[F(1,9)=9.52, p=.013]$, showing once again that the singleton effect with low working memory load $[t(9)=4.8, p<.001$, one tailed $]$ was increased with high working memory load, as we predicted (Figure 3 ). These results confirm that load on working memory per se increases attentional capture.

\section{GENERAL DISCUSSION}

The present results demonstrate that attentional capture by an irrelevant color singleton distractor during search for a target shape depends on whether working memory is available to the search task or is loaded with an unrelated working memory task. These findings provide support for the hypothesis that working memory provides goaldirected control of visual selective attention. Previous evidence for this hypothesis was confined to Stroop-like tasks, in which working memory load was found to determine both distractor congruency effects on behavior and neural responses related to the distractor (see, e.g., de Fockert et al., 2001; Lavie et al., 2004). Stroop-like tasks measure distractor interference via effects of response congruency on target RTs, whereas visual search tasks measure atten- tional capture via the RT cost produced by the presence of an irrelevant singleton. As such, the convergence of these different measures on the same conclusion suggests a general role for working memory in goal-directed control of visual selective attention that minimizes interference by any goal-irrelevant distractors.

This conclusion can accommodate the findings from a recent functional imaging study (de Fockert, Rees, Frith, \& Lavie, 2004). Using the same attentional capture task as was used here, de Fockert et al. (2004) found that the magnitude of singleton interference effects on behavior was negatively correlated with the level of activity in the left precentral gyrus of the frontal cortex, an area that was also associated with the main effect of working memory load in de Fockert et al. (2001). The finding of a negative correlation between frontal activity and singleton interference implies that top-down control functions mediated by frontal cortex prevent interference by the irrelevant singleton, a finding that is in line with our present demonstration of a direct role for working memory in the control of attentional capture.

The present findings also shed some light on the issue of whether attentional capture is purely stimulus driven or possibly subject to top-down control. The finding that attentional capture is modulated by working memory load clearly demonstrates that it is subject to top-down control. ${ }^{3}$ The finding, however, that an irrelevant singleton captures attention, even when top-down control functions are not loaded (as in the no-load or low-load conditions), points to a stimulus-driven component of attentional capture. The features we used for the target and for the singleton distractor meant that the irrelevant color singleton was more salient than the target shape. Under these conditions, the singleton intrudes into the search even when irrelevant and even when subjects had working memory capacity available to control against interference from the irrelevant distractor.

Our findings that working memory load increases capture by a salient singleton distractor during search con- 
trasts with previous findings that failed to find effects of working memory load on nontargets' rejection in search tasks that do not include any particularly salient competing distractor. This contrast supports our hypothesis that cognitive control of visual search by working memory is needed only in competitive situations in which a highpriority target competes for attention with a low-priority, but salient, distractor.

\section{REFERENCES}

Bacon, W. F., \& Egeth, H. E. (1994). Overriding stimulus-driven attentional capture. Perception \& Psychophysics, 55, 485-496.

BADDELEY, A. D. (1996). Exploring the central executive. Quarterly Journal of_Experimental Psychology, 49A, 1-28.

de Fockert, J. W., Rees, G., Frith, C. D., \& LAVIe, N. (2001). The role of working memory in visual selective attention. Science, 291, 1803-1806.

de Fockert, J. W., Rees, G., Frith, C. D., \& Lavie, N. (2004). Neural correlates of attentional capture in visual search. Journal of Cognitive Neuroscience, 16, 751-759.

DESIMONE, R., \& Duncan, J. (1995). Neural mechanisms of selective visual attention. Annual Review of Neuroscience, 18, 193-222.

DunCAN, J., \& HuMPhrEYS, G. W. (1989). Visual search and stimulus similarity. Psychological Review, 96, 433-458.

Engle, R. W., Conway, A. R. A., Tuholski, S. W., \& Shisler, R. J. (1995). A resource account of inhibition. Psychological Science, 6, 122-125.

KANE, M. J., \& Engle, R. W. (2003). Working-memory capacity and the control of attention: The contributions of goal neglect, response competition, and task set to Stroop interference. Journal of Experimental Psychology: General, 132, 47-70.

LAVIE, N. (2000). Selective attention and cognitive control: Dissociating attentional functions through different types of load. In S. Monsell \& J. Driver (Eds.), Control of cognitive processes: Attention and performance XVIII (pp. 175-194). Cambridge, MA: MIT Press.

Lavie, N., Hirst, A., DE Fockert, J. W., \& Viding, E. (2004). Load theory of selective attention and cognitive control. Journal of Experimental Psychology: General, 133, 339-354.

LogAN, G. D. (1978). Attention in character-classification tasks: Evidence for the automaticity of component stages. Journal of Experimental Psychology: General, 107, 32-63.
Theeuwes, J. (1992). Perceptual selectivity for color and form. Perception \& Psychophysics, 51, 599-606.

TheEUWES, J. (1996). Perceptual selectivity for color and form: On the nature of the interference effect. In A. Kramer, M. Coles, \& G. Logan (Eds.), Converging operations in the study of selective visual attention (pp. 297-314). Washington, DC: American Psychological Association.

Woodman, G. F., \& LucK, S. J. (2004). Visual search is slowed when visuospatial working memory is occupied. Psychonomic Bulletin \& Review, 11, 269-274.

Woodman, G. F., Vogel, E. K., \& LucK, S. J. (2001). Visual search remains efficient when visual working memory is full. Psychological Science, 12, 219-224.

YANTIS, S. (2000). Control of visual attention. In S. Monsell \& J. Driver (Eds.), Control of cognitive processes: Attention and performance XVIII (pp. 73-104). Cambridge, MA: MIT Press.

\section{NOTES}

1. For brevity's sake, we restrict our review to visual selective attention studies characterized by the need to ignore irrelevant distractors, as these are most relevant to our hypothesis about the role of working memory in the rejection of task-irrelevant distractors.

2. Note that the inclusion of an irrelevant singleton in search tasks does not eliminate the substantial differences between search and Stroop-like paradigms. For instance, target selection requires spatial search in search tasks, but not in Stroop-like tasks. The distractor pops out because of a salient feature in the search plus singleton tasks and therefore competes with the target during search, whereas the Stroop distractor is associated with a target response and therefore competes for response selection. Generalizing effects across these different tasks that involve different types of competition between target and distractors can thus provide strong convergent support for a general role of working memory in selective attention.

3. Note that the increased singleton effects cannot be attributed to reduced target identification with high working memory load. As can be seen in Figures 1-3, target RTs in the singleton-absent conditions showed little or no increase at all by high working memory load. It is safe to conclude that high working memory load increased capture by an irrelevant singleton. We thank Bruce Milliken for raising this issue.

(Manuscript received February 12, 2004; revision accepted for publication September 19, 2004.) 